\title{
Modelling of Sub-grid Scale Reaction Rate Based on a Novel Series Model: Application to a Premixed Bluff-Body Stabilised Flame
}

\author{
Weilin Zeng a , Konstantina Vogiatzaki b , Salvador Navarro-Martinez ${ }^{\mathrm{c}}$, and Kai Hong \\ Luo a,* \\ ${ }^{a}$ Department of Mechanical Engineering, University College London, Torrington Place, \\ London WC1E 7JE, UK \\ b Advanced Engineering Centre, University of Brighton, Lewes Road, Brighton, BN2 4AT, \\ UK \\ ${ }^{\mathrm{c}}$ Department of Mechanical Engineering, Imperial College, South Kensington Campus, \\ London SW7 2AZ, UK \\ * Corresponding Author: Kai Hong Luo \\ Postal Address: Department of Mechanical Engineering, University College London, \\ Torrington Place, London WC1E 7JE, UK \\ Fax: +44 (0)2073880180 \\ Email: K.Luo@ucl.ac.uk
}

\begin{abstract}
In this paper, a new model for closing the sub-grid reaction rate is proposed based on the series expansion of the chemical source term around the filtered value. For validation, large eddy simulations of a bluff-body stabilised premixed flame are performed at three different grid resolutions, and results are compared with experimental data. Simulations neglecting the sub-grid contributions of the source term are also conducted to examine the relative sub-grid contribution. The results show that the series model reproduces correctly key characteristics such as flame anchoring, recirculation zones and shear layers. Statistically, good agreement
\end{abstract}


with experimental data is obtained by the series model, in terms of time-averaged profiles of velocity and its fluctuations, and temperature as well as the size of the recirculation region. With increasing mesh refinement, the "no-model" approach results improve and the predictions are similar (albeit always worse) to those of the series model.

Keywords: Series model; Large Eddy Simulation; Sub-grid Scale Reaction Rate; Premixed Combustion

\section{Introduction}

Premixed turbulent combustion is arguably the most difficult regime to model for large eddy simulation (LES), as flame scales and flow scales can be widely different. An even more challenging case is premixed turbulent combustion involving solid walls. However, a common method in premixed combustors and laboratory burners to stabilise a turbulent flame is by means of flame holders such as a bluff body, where a recirculation zone of hot products is established to continuously ignite the mixture flows. A key to designing low-emission combustors and burners is a better understanding of combustion dynamics, including ignition, vortex shedding, turbulence-kinetics interaction, and flame-wall interactions including flameholding. The operation of such devices is often impaired by potentially harmful combustion instabilities, which at the lean limit may cause blow off, and at the rich limit may lead to flashback (Fureby, 2000a). In combustors with bluff-body flame holders, vortex shedding from a shear layer plays a significant role in flame anchoring due to the Kelvin-Helmholtz instability. Although research in this area has been active for over five decades, a thorough fundamental understanding of the relevant phenomena is still lacking, due to the difficulties in conducting spatially and temporally resolved experiments and numerical simulations. In particular, the 
non-linear interactions between the vortex shedding, heat release and volumetric expansion in the wake (Zettervall et al., 2017) present special difficulties, which often lead to thermoacoustic instabilities in unsteady flames.

The bluff-body stabilised premixed flame experiment with a rectangular cross-section and a triangular bluff-body performed under the Volvo Flygmotor AB program (Sjunnesson et al., 1991a, Sjunnesson et al., 1991b) has been simulated by many researchers for model validation and lean premixed combustion study. The most used combustion models for reproducing the bluff-body flame dynamics can be categorised into three groups. The first type is geometric approaches, which is based on flame-front geometry/topology using the flamelet assumption describing the flame as a front much thinner than any other length scale, and employing an effective flame surface to account for flame-turbulence interaction (Giacomazzi et al., 2004). Fureby (Fureby, 2000b) presented the development and application of a flamewrinkling LES combustion model in which transport equations for a reaction coordinate, a modelled flame-wrinkling density and the laminar flame speed are derived, modelled and solved for, and Cocks (Cocks et al., 2015) also used the same progress variable type model to study the impact of numerics on the predictive capabilities of reacting flow LES. Erickson (Erickson and Soteriou, 2011) focused on the influence of reactant temperature on the dynamics of bluff body stabilized premixed flames with the flame-sheet model, while Sankaran (Sankaran et al., 2012) employed the same model and studied the key physics of flame blow off. Park and Ko (Park and Ko, 2011) presented the application of a dynamic G-equation model and temperature and velocity results match experiments well. Ghani (Ghani et al., 2015) put the dynamic thickened flame model in practice and confirms the capacity of high order LES to capture not only low-frequency oscillations but also high-order frequency transverse modes in combustion chambers. Ma (Ma et al., 2014, Ma et al., 2013) developed a new algebraic model 
for Favre-filtered Scalar Dissipation Rate based on the flame surface density model and validated it using this flame.

The second category is models based on turbulent mixing descriptions, constraining the effective reaction rate and describing it in terms of scalar dissipation rate (Giacomazzi et al., 2004). Giacomazzi (Giacomazzi et al., 2004) used the fractal model to discover the coupling of turbulence and chemistry, and the model assumes that chemical reactions take place only at the dissipative scales of turbulence near the so-called "fine structures" (eddy dissipation concept). Zettervall (Zettervall et al., 2017) compared the influence of reaction mechanism on flames with the Partially Stirred Reactor LES model, using two well-known global reaction mechanisms and a novel skeletal reaction mechanism, and found that the choice of the reaction mechanism does not significantly influence the instantaneous or time-averaged velocity, whereas the instantaneous and time-averaged species and temperature are influenced.

The third class is statistical methods based on single-point probability density functions (PDF) of scalar fields and geometrical flame surface analysis, describing diffusive processes through micro-mixing models that are independent of chemical reactions. Length scale effects are indirectly included, by introducing the pdf as a function of the scalar dissipation rate. Möller (Möller et al., 1996) compared an eddy-dissipation-kinetic model, a presumed pdf approach and MILES and revealed a sufficient level of accuracy for all first- and second-order statistical moments available. Jones (Jones et al., 2015) tested the Eulerian stochastic field method, and the results show very good agreement with the experimental data demonstrating the capability of the LES method coupled with the SGS-PDF method in representing premixed combustion in complex flame configurations. Gokulakrishnan (Gokulakrishnan et al., 2009) modelled the flame instability and blowout in bluff-body stabilised flames with the LES-PDF approach. 
The present study proposes a different LES combustion model for turbulent reacting flows, based on series expansion. The model is evaluated in a bluff-body stabilised premixed flame at three grid resolutions.

\section{A Series Model for SGS Reaction Rate}

The LES equations for mass, momentum, mixture fraction and total enthalpy are obtained by applying low-pass filtering to the instantaneous governing equations (Poinsot and Veynante, 2005). The unresolved SGS stress tensor is closed using dynamic one equation eddy model (Fureby et al., 1997) in this study. For the combustion modelling, a new series SGS approach is developed following a mathematical derivation, where the multiple-dimensional Taylor series expansion of the unfiltered chemical source term is introduced.

Historically, a similar notion called the sub-filter-scale (SFS) stress model has been brought forward by researchers from Stanford University (Katopodes et al., 2000b, Katopodes et al., 2000a) and the Taylor expansion is basically on the filtered velocity field. In detail, the unsolved velocity is presented by inverse succession of Taylor series expansion on the velocity field. The derivation of expanding is straightforward and can be shown to be a good approximation to the unresolved velocity field, at least in low Reynolds number flows. The mathematical expansion serves to close the NavierStokes equations by providing an expression for the sub-grid Reynolds stress. These evolution equations allow systematic evaluation of the relative contributions by advection, diffusion, dissipation, pressure, rotation, and stratification in the sub-filter-scale effects felt by the resolved components of the flow(Katopodes et al., 2000b). The model is compared with direct numerical simulation results and achieves good accuracy(Katopodes et al., 2000b). Later, the approach is used to simulate a neutral boundary layer flow over a rough wall and show excellent agreement with similarity theory logarithmic velocity profiles, a significant improvement over standard eddy-viscosity closures(Chow et al., 2005).

The same notion has also been wielded in the premixed combustion context. Domingo and Vervisch from Normandie Université developed a new approach to sub-grid scale modelling turbulent 
reacting flows(Domingo and Vervisch, 2015) and to evaluate topology-based sub-grid scale combustion models(Domingo and Vervisch, 2017) based on the SFS model. The concrete procedures are stated as: a deconvolution operator is employed from a simple numerical treatment of the LES signal. The inversion of a discrete filter is derived in physical space from a Taylor expansion of the well-defined filtering operation, leading to explicit or implicit inverse filters, which are directly applied to the threedimensional scalar signals over the LES grid. The non-linear terms, as the chemical sources, are then computed from the deconvoluted signals, to be filtered back over the LES mesh to advance the solution in time(Domingo and Vervisch, 2017, Domingo and Vervisch, 2015). A turbulent Bunsen flame was simulated to validate the accuracy of the model. In comparison of the SFS model, the main difference is that the Taylor expansion is applied on the scalar field rather than the velocity field.

In this study, other than the above models, the series expansion is performed in the scalar space around the filtered value. This is due to the highly non-linear formation of the chemical source term, which may vary sharply and is not differentiable in physical space. For simplicity, the formalism is presented first for a single reactive scalar (the extension to multiple variables will be shown in the following sections):

$$
\dot{\omega}(c) \approx \dot{\omega}(\bar{c})+\left.\frac{\partial \dot{\omega}}{\partial c}\right|_{c=\bar{c}} \delta c+\left.\frac{1 \partial^{2} \dot{\omega}}{2 \partial c^{2}}\right|_{c=\bar{c}}(\delta c)^{2}+\ldots(1)
$$

Where the species molar concentration $c=\frac{\rho Y}{W}, \mathrm{~W}$ is the molecular weight.

Inside this equation, the term $\delta c=(c-\bar{c})$ is hardly to be predicted in the frame of large eddy simulation. In this sense, a transformation is added to the above equation:

$$
\delta c=\frac{\partial c}{\partial x_{i}} \delta x_{i}=\frac{\partial c}{\partial x_{i}}\left(x_{i}-\overline{x_{i}}\right)
$$

The equation takes the form as:

$$
\begin{aligned}
& \dot{\omega}(c) \approx \dot{\omega}(\bar{c})+\left.\frac{\partial \dot{\omega}}{\partial c}\right|_{c=\bar{c}} \frac{\partial c}{\partial x_{i}} \delta x_{i}+\left.\frac{1 \partial^{2} \dot{\omega}}{2 \partial c^{2}}\right|_{c=\bar{c}}\left(\frac{\partial c}{\partial x_{i}} \delta x_{i}\right)^{2}+\ldots \\
& =\dot{\omega}(\bar{c})+\left.\left(x_{m}-\overline{x_{m}}\right) \frac{\partial \dot{\omega}}{\partial c}\right|_{c=\bar{c}} \frac{\partial c}{\partial x_{m}}+\left.\left(x_{m}-\overline{x_{m}}\right)\left(x_{n}-\overline{x_{n}}\right) \frac{1 \partial^{2} \dot{\omega}}{\partial c^{2}}\right|_{c=\bar{c}} \quad \frac{\partial c \partial c}{\partial x_{m} \partial x_{n}}+\ldots
\end{aligned}
$$


Note for compactness, index notation is used.

Now an anisotropic Gaussian filter or other filter is applied to the both side of the equation. All terms with odd powers of $\mathrm{x}, \mathrm{y}$, and $\mathrm{z}$ vanish as result of symmetry elimination(Chow et al., 2005, Katopodes et al., 2000a, Domingo and Vervisch, 2015, Domingo and Vervisch, 2017).

$$
\begin{aligned}
& \overline{\dot{\omega}(c)}=\dot{\omega}(\bar{c})+\left.\frac{\Delta_{x}^{2} \partial^{2} \dot{\omega}}{24 \partial c^{2}}\right|_{c=\bar{c}} \overline{\left(\frac{\partial c^{2}}{\partial x}\right)^{2}}+\left.\frac{\Delta_{y}^{2} \partial^{2} \dot{\omega}}{24 \partial c^{2}}\right|_{c=\bar{c}} \overline{\left(\frac{\partial c^{2}}{\partial y}\right)^{2}}+\left.\frac{\Delta_{z}^{2} \partial^{2} \dot{\omega}}{24 \partial c^{2}}\right|_{c=\bar{c}} \overline{\left(\frac{\partial c^{2}}{\partial z}\right)^{2}}+ \\
& \left.\frac{\Delta_{x}^{4} \partial^{4} \dot{\omega}}{1152 \partial c^{4}}\right|_{c=\bar{c}} \overline{\left(\frac{\partial c^{4}}{\partial x}\right)}+\left.\frac{\Delta_{y}^{4} \partial^{4} \dot{\omega}}{1152 \partial c^{4}}\right|_{c=\bar{c}} \overline{\left(\frac{\partial c^{4}}{\partial y}\right)^{4}}+\left.\frac{\Delta_{z}^{4} \partial^{4} \dot{\omega}}{1152 \partial c^{4}}\right|_{c=\bar{c}} \overline{\left(\frac{\partial c^{4}}{\partial z}\right)^{4}}+\left.\frac{\Delta_{x}^{2} \Delta_{y}^{2} \partial^{4} \dot{\omega}}{1728 \partial c^{4}}\right|_{c=\bar{c}} \overline{\left(\frac{\partial c^{2}}{\partial x}\right)^{2}\left(\frac{\partial c^{2}}{\partial y}\right)^{2}}+ \\
& \left.\frac{\Delta_{x}^{2} \Delta_{z}^{2} \partial^{4} \dot{\omega}}{1728 \partial c^{4}}\right|_{c=\bar{c}} \overline{\left(\frac{\partial c^{2}}{\partial x}\right)\left(\frac{\partial c^{2}}{\partial z}\right)^{2}}+\left.\frac{\Delta_{z}^{2} \Delta_{y}^{2} \partial^{4} \dot{\omega}}{1728 \partial c^{4}}\right|_{c=\bar{c}} \overline{\left.\frac{\partial c^{2}}{\partial z}\right)^{2}\left(\frac{\partial c^{2}}{\partial y}\right)^{2}}+O\left(\Delta^{6}\right)
\end{aligned}
$$

When the filter (like the top-hat filter in this study) is isotropic, the equation is arranged as:

$$
\begin{aligned}
& \overline{\dot{\omega}(c)}=\dot{\omega}(\bar{c})+\left.\frac{\Delta^{2} \partial^{2} \dot{\omega}}{24 \partial c^{2}}\right|_{c=\bar{c}}\left[\overline{\left(\frac{\partial c}{\partial x}\right)^{2}}+\overline{\left(\frac{\partial c}{\partial y}\right)^{2}}+\overline{\left(\frac{\partial c}{\partial z}\right)^{2}}\right]+\left.\frac{\Delta^{4} \partial^{4} \dot{\omega}}{1152 \partial c^{4}}\right|_{c=\bar{c}}\left[\overline{\left(\frac{\partial c}{\partial x}\right)^{4}}+\overline{\left(\frac{\partial c}{\partial y}\right)^{4}}+\overline{\left(\frac{\partial c}{\partial z}\right)^{4}}\right]+ \\
& \left.\frac{\Delta^{4} \partial^{4} \dot{\omega}}{1728 \partial c^{4}}\right|_{c=\bar{c}}\left[\overline{\left(\frac{\partial c}{\partial x}\right)^{2}\left(\frac{\partial c}{\partial y}\right)^{2}}+\overline{\left(\frac{\partial c}{\partial x}\right)^{2}\left(\frac{\partial c}{\partial z}\right)^{2}}+\overline{\left(\frac{\partial c}{\partial z}\right)^{2}\left(\frac{\partial c}{\partial y}\right)^{2}}\right]+O\left(\Delta^{6}\right)
\end{aligned}
$$

For turbulent scalar signals, the fourth order terms were not found to play a major role, and the filtering may be achieved with only the second-order derivatives(Katopodes et al., 2000a). Such approximate filtering has already been used in the atmospheric boundary layer scalar transport(Chow et al., 2005) and also combustion context, to perform a priori tests of SGS modelling from the filtering of Direct Numerical Simulation (DNS)(Moureau et al., 2011).Terms of $\mathrm{O}\left(\Delta^{4}\right)$ and higher are neglected. Numerical schemes for scalar gradients in LES of reactive flows are often second order and therefore retaining the terms $\mathrm{O}\left(\Delta^{4}\right)$ the series model will only be sixth-order accurate if the order of the overall scheme will change to the same order. The final expressions is therefore:

$$
\overline{\dot{\omega}(c)}=\dot{\omega}(\bar{c})+\left.\frac{\Delta^{2} \partial^{2} \dot{\omega}}{24 \partial c^{2}}\right|_{c=\bar{c}} \overline{\left(\frac{\partial c}{\partial x_{i}}\right)^{2}}+O\left(\Delta^{4}\right)
$$


The same procedures can be applied to the source term, which is a function of multiple species, temperature and pressure:

$$
\begin{aligned}
& \overline{\omega_{\alpha}\left(\varphi_{1}, \varphi_{2}, \ldots, \varphi_{k}\right)}=\dot{\omega_{\alpha}}\left(\overline{\varphi_{1}}, \overline{\varphi_{2}}, \ldots, \overline{\varphi_{k}}\right)+
\end{aligned}
$$

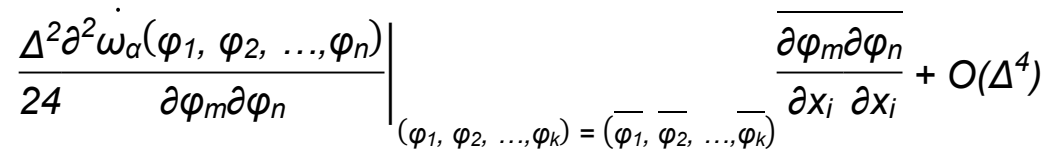

Inside the equation, the filtered scalar gradient term is not closed. Similarly, it takes the form of scalar dissipation term:

$$
\overline{\frac{\partial \varphi_{m} \partial \varphi_{n}}{\partial x_{i} \partial x_{i}}}=\frac{\overline{\partial \varphi_{m}} \partial \overline{\varphi_{n}}}{\partial x_{i} \partial x_{i}}+X s g s
$$

Where $X_{\text {sgs }}$ behaves in analogy to a sub-grid scalar dissipation rate, and it accounts for the effects of un-resolved scalar gradients. In the present work, an algebraic approach (Knudsen et al., 2012) is employed as:

$$
X_{s g s}=C_{s g s} \frac{\partial \overline{\varphi_{m}} \partial \overline{\varphi_{n}}}{\partial x_{i} \partial x_{i}}
$$

In the context of non-premixed combustion, $C_{s g s}$ is widely chosen to be 0.1 following (NavarroMartinez and Kronenburg, 2007, Branley and Jones, 2001). In this study, $C_{s g s}=0$ is presumed for lack of relevant empirical values reported in previous premixed flame research. Note that this assumption can be inaccurate if the flames are poorly resolved. Another method to model the scalar dissipation type term is transport equation models, as (Knudsen et al., 2012) proposed and tested on a non-premixed auto-ignition jet flame. The results showed better accuracy over the algebraic approach. However, its employment remains within non-premixed scopes. The application to premixed regimes will be explored in future studies.

In this way, the model is explicitly closed for premixed combustion as:

$$
\overline{\omega_{\alpha}\left(\varphi_{1}, \varphi_{2}, \ldots, \varphi_{k}\right)}=\dot{\omega_{\alpha}}\left(\overline{\varphi_{1}}, \overline{\varphi_{2}}, \ldots, \overline{\varphi_{k}}\right)+
$$




$$
\left.\frac{\Delta^{2} \partial^{2} \dot{\omega}_{\alpha}\left(\varphi_{1}, \varphi_{2}, \ldots, \varphi_{n}\right)}{\partial 4}\right|_{\left(\varphi_{1}, \varphi_{2}, \ldots, \varphi_{k}\right)=\left(\overline{\varphi_{1}}, \overline{\varphi_{2}}, \ldots, \overline{\varphi_{k}}\right)} \frac{\partial \overline{\varphi_{m}} \partial \overline{\varphi_{n}}}{\partial x_{i} \partial x_{i}}+O\left(\Delta^{4}\right)(10)
$$

The series model can be understood as a combination of a chemical source term neglecting SGS terms (a no-model or perfectly mixed closure) and a SGS contribution which depends on the square of the filter width:

$$
\overline{\omega_{\alpha}\left(\varphi_{1}, \varphi_{2}, \ldots, \varphi_{k}\right)}=\dot{\omega_{\alpha}}\left(\overline{\varphi_{1}}, \overline{\varphi_{2}}, \ldots, \overline{\varphi_{k}}\right)+\dot{\omega_{s g s}}
$$

The first term in the above equation is just the Arrhenius expression of the particular chemical mechanism, while to evaluate $\dot{\omega}_{\text {sgs }}$, the second derivatives of the chemical source term are needed, which can be analytically obtained by:

$$
\left.\frac{\partial^{2} \dot{\omega}}{\partial c^{2}}\right|_{c=\bar{c}}=\frac{\partial^{2} \dot{\omega}_{\alpha}}{\partial c_{\alpha}{ }^{2}}=W_{\alpha} \sum_{j=1}^{N R}\left(v_{f}^{\alpha j}-v_{b}^{\alpha j}\right)\left(k_{f}^{j} v_{f}^{\alpha j}\left(v_{f}^{\alpha j}-1\right) c_{\alpha}^{v_{f}^{\alpha j}}-2 \prod_{\substack{i=1 \\ i \neq \alpha}}^{N S} c_{i}^{v_{j}^{i j}}-k_{b}^{j} v_{b}^{\alpha j}\left(v_{b}^{\alpha j}-1\right) c_{\alpha}^{v_{b}^{\alpha j}}-2 \prod_{\substack{i=1 \\ i \neq \alpha}}^{N S} c_{i}^{v_{i j}^{i j}}\right)
$$

In complex mechanisms, the second-derivatives could be pre-computed and tabulated (Auzillon et al., 2012, Bekdemir et al., 2013, Kim and Pope, 2014). The scalar gradients in Eq. (9) are obtained directly from the flow field. Forward and backward rates are expressed in the form of Arrhenius laws and therefore finite-rate chemistry effects are directly included at both resolved and unresolved scales.

As the full expression of the series closure is arrived at, its unique features different than the conventional models can be summarised. First, the series model is a mathematical approach. It has no assumption regarding the combustion regime and, a-priori, the model could be applied to different combustion regimes such as premixed, non-premixed, and partially premixed combustion. Second, although similar approaches (Villasenor et al., 1992) had been tried within the RANS context, they were not successful. In RANS the non-linear terms of the series expansion $\left(\omega_{\text {fluctuation }}\right)$ can have values much higher than the first order term, because of the large temporal fluctuations. This makes the model very sensitive to the modelling of the terms $\left(\overline{\mathrm{Y}_{\mathrm{F}} \mathrm{Y}_{\mathrm{O}} "}, \overline{\mathrm{Y}_{\mathrm{F}}{ }^{\prime \prime}}\right)$. In LES, on the contrary, the magnitude of this term is smaller given a relatively well-resolved grid that is always necessary to capture (non-reactive) flow characteristics related to features above the Taylor scale. Besides, the accuracy level of the method 
is determined by the Taylor series order. Last but not least, the model also predicts the correct limiting behaviour, and the sub-grid contribution reduces with the square of the filter size, approaching DNS as $\Delta$ approaches Kolmogorov scales.

\section{Test Case Description}

The case under consideration is the bluff-body stabilised premixed flame experiment performed under the Volvo Flygmotor AB program. It is relatively simple but includes many features in practical combustors, such as flame anchoring, recirculation zones, and shear layers. It has been used for LES model validation (Baudoin et al., 2009, Jones et al., 2015, Ma et al., 2014, Wang et al., 2011, Emerson et al., 2011, Ma et al., 2013) and lean premixed combustion study (Zettervall et al., 2017, Erickson and Soteriou, 2011, Cocks et al., 2015, Fureby, 2000a, Kiel et al., 2007, Porumbel and Menon, 2006, Kim and Pope, 2014, Shanbhogue et al., 2009, Ghani et al., 2015). The configuration consists of a $1 \mathrm{~m}$ long straight channel with a rectangular cross-section of $0.12 \times 0.24 \mathrm{~m}$. The flame is anchored on a bluff body, having an equilateral triangular cross-section (side length $H=0.04 \mathrm{~m}$ ), located $0.68 \mathrm{~m}$ upstream of the exit. A propane and air mixture at $1 \mathrm{~atm}$ and $288 \mathrm{~K}\left(T_{i n}\right)$ is introduced at an equivalence ratio of 0.65 . The mixture flows at a bulk inlet velocity of $U_{\text {in }}=17 \mathrm{~m} / \mathrm{s}$, resulting in a bulk Reynolds number of $R e=U_{i n} H / v=48000$ and a Karlovitz number of $K a=\delta_{L}^{2} / \eta^{2}=62$, with an inlet turbulence intensity of $3-4 \%$. It is in the thin reactions zone regime. In the experiment, temperature was measured by CARS and velocity by LDA (Sjunnesson et al., 1991a).

The grid size is chosen based on two criteria: the resolution of the boundary layer on the bluff body, estimated to be around $5 \mathrm{~mm}$ (Cocks et al., 2015), and the Pope energy criterion (Pope, 2001) which suggests that a filter-width to integral length scale ratio of $\Delta / H=0.083$ should be maintained to resolve $80 \%$ of the turbulent kinetic energy. The latter criterion gives 
a maximum filter width of $3.3 \mathrm{~mm}$. Three grid resolutions of 3, 2 and $1 \mathrm{~mm}$. Respectively, are generated, where the filtered width is the cubic root of the cell volume. The grids employed are nearly isotropic $(\Delta x=\Delta y=\Delta z)$ in most regions except near walls, where grids are stretched to capture near-wall motions. All simulations are performed on grids with a span-wise depth of $4 \mathrm{H}$, with periodic boundary conditions. The domain accounts for the combustor section extending $2.5 H$ upstream and $17 H$ downstream of the flame-holder trailing edge. The total cell varies from 0.56 million in the coarse mesh to 15.1 million in the $1 \mathrm{~mm}$ grid. At the inlet, Dirichlet conditions are used for all variables except pressure, for which zero Neumann conditions are employed. At the exit, zero Neumann conditions are specified for all variables except pressure, for which wave-transmissive conditions are used. No-slip conditions are applied to walls of the duct as well as the bluff-body, while zero Neumann conditions are specified for the other variables. The time-step is variable, and the compressible Courant number is lower than 0.2 throughout the simulations. Chemistry is computed using a singlestep mechanism for propane/air flames (Peters and Rogg, 2008). Previous comparisons (Zettervall et al., 2017) showed that the choice of the reaction mechanism (simplified or detailed) does not significantly influence the instantaneous or time-averaged velocity, and simplified mechanisms (Cocks et al., 2015, Giacomazzi et al., 2004, Jones et al., 2015) have achieved good predictions in this configuration. Simulations with the "no-model" approach (excluding the SGS effect, $\overline{\omega_{\alpha}\left(\varphi_{1}, \varphi_{2}, \ldots, \varphi_{k}\right)}=\dot{\omega_{\alpha}}\left(\overline{\varphi_{1}}, \overline{\varphi_{2}}, \ldots, \overline{\varphi_{k}}\right)$ ), are also employed to examine the influence of the SGS contribution..

The series model is implemented into the finite-volume open-source solver OpenFOAM (Weller et al., 1998) to conduct LES with the low Mach number assumption. The Pimple algorithm is used for the velocity-pressure coupling and a second order implicit CrankNicholson scheme for time marching. The convective fluxes are reconstructed using multidimensional cell limited linear interpolation, while diffusive fluxes are reconstructed using a 
combination of central difference approximations and gradient face interpolation(Zettervall et al., 2017). Statistical collection is performed over 8 burner flow-through times. Prior to that, 6 burner flow-through times are simulated to ensure the flow is established.

\section{Results and Discussions}

\subsection{Instantaneous flow-field structures}

In this part, results are presented for the series model in $2 \mathrm{~mm}$ resolution grids. Figure 2 shows typical vortical structures after the bluff-body, represented by iso-surfaces of vorticity magnitude. The large-scale coherent vortices are shed from the shear layer due to KelvinHelmholtz instabilities, which break down into smaller scale eddies downstream. A von Karman vortex street is established in the wake of the body characterised by nearly symmetric vortex shedding. Observing the temperature distribution in Figure. 3, hot combustion products inside the recirculation zone incessantly mix with the cold co-flowing mixture, and sequentially ignition occurs in the shear/mixing layers. The ignited flame convects downstream and continues to ignite the neighbouring mixtures by heat transfer. The recirculation region behind the flame-holder, sustaining this continuous re-ignition process, stabilises the flame. The series model correctly reproduces the flame-anchoring features.

Figure 3 depicts the contours of temperature, the reaction rate of $\mathrm{C}_{3} \mathrm{H}_{8}$ and its SGS part. Identical to vortex shedding in Figure 2, flame propagation is presented almost symmetrically at least before $x / H=6$. This behaviour was also reported in previous LES (Cocks et al., 2015, Zettervall et al., 2017, Möller et al., 1996). The chemical reaction takes place in the shear layer between the wake and unburnt mixtures. This reacting zone is identified by the reaction rate of $\mathrm{C}_{3} \mathrm{H}_{8}$. The SGS contribution to the source term oscillates between $-6 \%$ and $18 \%$, and it appears mostly in the reaction zone accordingly, where scalar gradients are large and SGS 
fluctuations are expected to play a major role. Large SGS contributions first appear around $x / H$ $=3.7$ in Figure 3 (c) in the shear layer, probably due to vortex shedding in the recirculation zone that enhances the turbulence intensity and mixing. Further downstream at $x / H=12$, strong SGS levels show up again, where small scale eddies roll up and merge (see Figure 2), indicating important sub-grid turbulence-chemistry interactions.

\subsection{Statistical flow-field results}

Distributions of mean and RMS axial velocities between the no model approach and series model at three different grid resolutions are presented in Figure 4. The iso-contour lines are outlined in the mean profile, while the streamlines of mean velocity are plotted in RMS. Both models show that the mean flow is dominated by a recirculation zone (indicated by negative axial velocity) behind the flame-holder and strong shear layers originating from the bluff-body corners. The series model predicts a larger recirculation region (from $x / H=3.4$, until 3.1) for the fine and coarse meshes, respectively. The recirculation region ranges from $x / H=3.1$, to 2.7 in the no-model approach. In conjunction with the centreline profile in Figure 5, the experimental recirculation length is $3.5 \mathrm{H}$, showing that the series model predicts a recirculation length very close to experimental measurements. Accurate predictions of this region are a key to simulating bluff-body-like combustors as it plays a vital role in anchoring flames and periodically mixing reactants and products. As to RMS profiles, both models predict a lower fluctuation level when the grid is refined. The difference between models is obvious in the coarsest $(3 \mathrm{~mm})$ resolution.

Comparisons of simulated and experimentally measured time-averaged axial velocity profiles along four axial sections $(x / H=0.375,1.63$, and 3.75 in the recirculation zone, $x / H=$ 9.4 in the downstream, these locations are outlined by white dash lines in Figure 4.) through 
the burner are shown in Figure 6. Overall, a reasonably good agreement with experimental data is achieved by the new series model, even at the coarsest resolutions. The model correctly reproduces the velocity transition from U-shape near the bluff body to the V-shape at the end of the recirculation zone and the flat profile in the downstream wake. Overall, all models slightly over-estimate the mean velocity downstream. However, the series model gives the closest agreement with experimental data. It could be related to the more intense volumetric thermal expansion (Ma et al., 2014) simulated in these locations. In Figure 7, the predicted mean temperatures are compared with experimental data. Despite the series model producing the best agreement with experimental data, the peak temperature is slightly over-predicted, which might account for the acceleration prediction downstream. Besides, the overall trends in the RMS velocity are well captured, with two separate peaks located at the shear layers in the recirculation zone. However, the no-model predicts over 3 times as large RMS fluctuations as experimental measurements downstream; the series model result gives less adequate RMS fluctuation peaks at $\mathrm{x} / \mathrm{H}=1.63$. The small discrepancies could be attributed to the simplicity of the chemical mechanism in use. Nevertheless, the series model also over-predicts experimental fluctuations downstream but provides good agreement within the recirculation region. It also demonstrates good predictive abilities of the simplified chemistry incorporated into the series model.

Comparing the series model in different grid resolutions, the 1 and $2 \mathrm{~mm}$ results are closer, especially for the mean and RMS profile at $x / H=3.75$ where the $3 \mathrm{~mm}$ results show a more obvious departure from the formers. As expected, despite neglecting sub-grid fluctuations, the no-model results improve as the grid resolution increases, although the predictions remain worse than those of the series model. If the grid is further refined, $\Delta \rightarrow 0$ and $\dot{\omega}_{s g s} \rightarrow 0$, and all models are expected to converge to a DNS solution, since the SGS terms should tend to zero. The above grid sensitivity analysis confirms the correct limiting behaviour of the series model. 


\section{Conclusions}

A new model for closing the sub-grid reaction rate is proposed based on series expansion of the chemical source term around the filtered value. For the purpose of validation, LES simulations of a bluff-body stabilized premixed flame are performed at three different grid resolutions, and results are compared with experimental data. Simulations neglecting the subgrid contributions of the source term are also carried out to examine the relative sub-grid contribution. The results show that the series model reproduces correctly key characteristics such as flame anchoring, recirculation zones and shear layers. Statistically, good agreement with experimental data is obtained by the series model, in terms of time-averaged profiles of velocity and its fluctuations, and temperature as well as the size of the recirculation region. In the finest mesh, the "no-model" approach results improve and predictions are similar (albeit always worse) to the series model.

For future study, the series model will be applied on a wider range of different inlet conditions to explore its predictive capability under different Reynolds and Karlovitz numbers. 


\section{Nomenclature}

\begin{tabular}{|c|c|c|c|}
\hline$\alpha$ & chemical species & $W$ & molecular weight \\
\hline$\chi$ & scalar dissipation rate & $c$ & species mass concentration \\
\hline$\dot{\omega}(c)$ & chemical source term & $x_{i}$ & spatial coordinate in $i$-direction \\
\hline$\rho$ & density & $\Delta$ & filter size \\
\hline$v_{f}^{\alpha j}$ & $\begin{array}{l}\text { molar stoichiometric coefficient of } \\
\text { species } \alpha \text { in reaction } j \text { (left) }\end{array}$ & $\varphi_{k}$ & field scalar \\
\hline$v_{b}^{a j}$ & $\begin{array}{l}\text { molar stoichiometric coefficient of } \\
\text { species } \alpha \text { in reaction } j \text { (right) }\end{array}$ & $C_{s g s}$ & sub-grid coefficient \\
\hline$k_{f}^{j}$ & $\begin{array}{l}\text { the rate coefficient for the forward } \\
\text { reaction coefficients }\end{array}$ & $Y$ & species mass fraction \\
\hline$k_{b}^{j}$ & $\begin{array}{l}\text { the rate coefficient for the backward } \\
\text { reaction coefficients }\end{array}$ & $\operatorname{Re}$ & Reynolds number \\
\hline$H$ & side length & & inlet bulk velocity \\
\hline$v$ & the kinematic viscosity & $K a$ & Karlovitz number \\
\hline$\delta_{L}$ & the laminar flame speed & $\eta$ & the flame thickness \\
\hline
\end{tabular}

\section{Acknowledgements}

This work was funded by the UK Engineering and Physical Sciences Research Council under the project "High Performance Computing Support for United Kingdom Consortium on Turbulent Reacting Flow (UKCTRF)" (Grant Nos. EP/K024876/1 and EP/R029369/1). Dr Vogiatzaki would like to acknowledge UK Engineering and Physical Science Research Council support through the grant EP/P012744/1. Sponsorships for Weilin Zeng from University College London (Dean`s Prize) and China Scholarship Council are also gratefully acknowledged. 


\section{References}

AUZILLON, P., GICQUEL, O., DARABIHA, N., VEYNANTE, D. \& FIORINA, B. 2012. A filtered tabulated chemistry model for LES of stratified flames. Combustion and flame, 159, 2704-2717.

BAUDOIN, E., YU, R., NOGENMYR, K.-J., BAI, X.-S. \& FUREBY, C. Comparison of LES models applied to a bluff body stabilized flame. 47th AIAA Aerospace Sciences Meeting, 2009. AIAA.

BEKDEMIR, C., SOMERS, L., DE GOEY, L., TILLOU, J. \& ANGELBERGER, C. 2013. Predicting diesel combustion characteristics with large-eddy simulations including tabulated chemical kinetics. Proceedings of the Combustion Institute, 34, 3067-3074.

BRANLEY, N. \& JONES, W. 2001. Large eddy simulation of a turbulent non-premixed flame. Combustion and Flame, 127, 1914-1934.

CHOW, F. K., STREET, R. L., XUE, M. \& FERZIGER, J. H. 2005. Explicit filtering and reconstruction turbulence modeling for large-eddy simulation of neutral boundary layer flow. Journal of the Atmospheric Sciences, 62, 2058-2077.

COCKS, P. A., SOTERIOU, M. C. \& SANKARAN, V. 2015. Impact of numerics on the predictive capabilities of reacting flow LES. Combustion and Flame, 162, 3394-3411.

DOMINGO, P. \& VERVISCH, L. 2015. Large Eddy Simulation of premixed turbulent combustion using approximate deconvolution and explicit flame filtering. Proceedings of the Combustion Institute, 35, 1349-1357.

DOMINGO, P. \& VERVISCH, L. 2017. DNS and approximate deconvolution as a tool to analyse one-dimensional filtered flame sub-grid scale modelling. Combustion and Flame, 177, 109-122.

EMERSON, B., LUNDRIGAN, J., O'CONNOR, J., NOBLE, D. \& LIEUWEN, T. Dependence of the bluff body wake structure on flame temperature ratio. 49th AIAA Aerospace Sciences Meeting including the New Horizons Forum and Aerospace Exposition, 2011. 597.

ERICKSON, R. \& SOTERIOU, M. 2011. The influence of reactant temperature on the dynamics of bluff body stabilized premixed flames. Combustion and Flame, 158, 2441-2457.

FUREBY, C. 2000a. A computational study of combustion instabilities due to vortex shedding. Proceedings of the Combustion Institute, 28, 783-791. 
FUREBY, C. 2000b. Large eddy simulation of combustion instabilities in a jet engine afterburner model. Combustion science and technology, 161, 213-243.

FUREBY, C., TABOR, G., WELLER, H. \& GOSMAN, A. 1997. A comparative study of subgrid scale models in homogeneous isotropic turbulence. Physics of fluids, 9, 1416-1429.

GHANI, A., POINSOT, T., GICQUEL, L. \& STAFFELBACH, G. 2015. LES of longitudinal and transverse self-excited combustion instabilities in a bluff-body stabilized turbulent premixed flame. Combustion and Flame, 162, 4075-4083.

GIACOMAZZI, E., BATTAGLIA, V. \& BRUNO, C. 2004. The coupling of turbulence and chemistry in a premixed bluff-body flame as studied by LES. Combustion and Flame, 138, 320335.

GOKULAKRISHNAN, P., FOLI, K., KLASSEN, M., ROBY, R., SOTERIOU, M., KIEL, B. \& SEKAR, B. LES-PDF Modeling of Flame Instability and Blow-Out in Bluff-Body Stabilized Flames. 45th AIAA/ASME/SAE/ASEE Joint Propulsion Conference \& Exhibit, 2009. 5409. JONES, W., MARQUIS, A. \& WANG, F. 2015. Large eddy simulation of a premixed propane turbulent bluff body flame using the Eulerian stochastic field method. Fuel, 140, 514-525.

KATOPODES, F. V., STREET, R. \& FERZIGER, J. Subfilter-scale scalar transport for large-eddy simulation. 14th Symposium on Boundary Layers and Turbulence, 2000a. American Meteorologic Society Aspen (CO), 472-475.

KATOPODES, F. V., STREET, R. L. \& FERZIGER, J. H. 2000b. A theory for the subfilter-scale model in large-eddy simulation. Environmental Fluid Mechanics Laboratory Tech. Rep, K1.

KIEL, B., GARWICK, K., GORD, J. R., MILLER, J., LYNCH, A., HILL, R. \& PHILLIPS, S. 2007. A detailed investigation of bluff body stabilized flames. AIAA Paper No. 2007-168, 168.

KIM, J. \& POPE, S. B. 2014. Effects of combined dimension reduction and tabulation on the simulations of a turbulent premixed flame using a large-eddy simulation/probability density function method. Combustion Theory and Modelling, 18, 388-413.

KNUDSEN, E., RICHARDSON, E., DORAN, E., PITSCH, H. \& CHEN, J. 2012. Modeling scalar dissipation and scalar variance in large eddy simulation: Algebraic and transport equation closures. Physics of Fluids, 24, 055103. 
MA, T., GAO, Y., KEMPF, A. M. \& CHAKRABORTY, N. 2014. Validation and implementation of algebraic LES modelling of scalar dissipation rate for reaction rate closure in turbulent premixed combustion. Combustion and Flame, 161, 3134-3153.

MA, T., STEIN, O., CHAKRABORTY, N. \& KEMPF, A. 2013. A posteriori testing of algebraic flame surface density models for LES. Combustion Theory and Modelling, 17, 431-482.

MÖLLER, S.-I., LUNDGREN, E. \& FUREBY, C. Large eddy simulation of unsteady combustion. Symposium (International) on Combustion, 1996. Elsevier, 241-248.

MOUREAU, V., DOMINGO, P. \& VERVISCH, L. 2011. From large-eddy simulation to direct numerical simulation of a lean premixed swirl flame: Filtered laminar flame-pdf modeling. Combustion and Flame, 158, 1340-1357.

NAVARRO-MARTINEZ, S. \& KRONENBURG, A. 2007. LES-CMC simulations of a turbulent bluff-body flame. Proceedings of the Combustion Institute, 31, 1721-1728.

PARK, N. S. \& KO, S. C. 2011. Large eddy simulation of turbulent premixed combustion flow around bluff body. Journal of mechanical science and technology, 25, 2227.

PETERS, N. \& ROGG, B. 2008. Reduced kinetic mechanisms for applications in combustion systems, Springer Science \& Business Media.

POINSOT, T. \& VEYNANTE, D. 2005. Theoretical and numerical combustion, RT Edwards, Inc. POPE, S. B. 2001. Turbulent flows, IOP Publishing.

PORUMBEL, I. \& MENON, S. 2006. Large eddy simulation of bluff body stabilized premixed flame. AIAA paper.

SANKARAN, V., PALIES, P., LILJENBERG, S., TEERLINCK, K. \& SOTERIOU, M. Stabilization dynamics of bluff-body premixed flames. 50th AIAA Aerospace Sciences Meeting including the New Horizons Forum and Aerospace Exposition, 2012. 352.

SHANBHOGUE, S. J., HUSAIN, S. \& LIEUWEN, T. 2009. Lean blowoff of bluff body stabilized flames: Scaling and dynamics. Progress in Energy and Combustion Science, 35, 98-120.

SJUNNESSON, A., NELSSON, C. \& MAX, E. 1991a. LDA measurements of velocities and turbulence in a bluff body stabilized flame. Laser Anemometry, 3, 83-90. 
SJUNNESSON, A., OLOVSSON, S. \& SJOBLOM, B. Validation rig- A tool for flame studies. International Symposium on Air Breathing Engines, 10 th, Nottingham, England, 1991b. 385393.

VILLASENOR, R., CHEN, J.-Y. \& PITZ, R. 1992. Modeling ideally expanded supersonic turbulent jet flows with nonpremixed H2-air combustion. AIAA journal, 30, 395-402.

WANG, G., BOILEAU, M. \& VEYNANTE, D. 2011. Implementation of a dynamic thickened flame model for large eddy simulations of turbulent premixed combustion. Combustion and Flame, $158,2199-2213$.

WELLER, H. G., TABOR, G., JASAK, H. \& FUREBY, C. 1998. A tensorial approach to computational continuum mechanics using object-oriented techniques. Computers in physics, 12 , $620-631$.

ZETTERVALL, N., NORDIN-BATES, K., NILSSON, E. \& FUREBY, C. 2017. Large Eddy Simulation of a premixed bluff body stabilized flame using global and skeletal reaction mechanisms. Combustion and Flame, 179, 1-22. 


\section{List of figures}

Figure 1. Schematic of the Volvo Rig combustor. The interior width in the z-direction is $6 H$.

Figure 2. Iso-surface of vorticity magnitude level $2000 \mathrm{~s}^{-1}$ vorticity colored by the $\mathrm{z}$ component of vorticity.

Figure 3. Instantaneous contours of $a$ ) temperature, $b$ ) absolute value of the $\mathrm{C}_{3} \mathrm{H}_{8}$ reaction rate $|\dot{\omega}(\bar{c})|$ and $c$ ) SGS contribution ratio of $\mathrm{C}_{3} \mathrm{H}_{8}$ reaction rate, $\dot{\omega} S G S / \dot{\omega}(\bar{c})$.

Figure 4. Mean (left) and RMS (right) axial velocity contours at different grid resolutions. $\mathrm{NM}$ is short for no model approach and SM for series model. $1 \mathrm{~mm}, 2 \mathrm{~mm}$, and $3 \mathrm{~mm}$ stand for the grid resolutions. These abbreviations are also in effect in the following figures. Black lines in the left part represent the iso-contours of different mean velocity levels in the colour bar. Black lines with arrows stand for the stream traces of the mean velocity field.

Figure 5. Centerline mean axial profile for different models in different grid resolutions

Figure 6. Mean and RMS axial velocity profiles for different models in different grid resolutions

Figure 7. Predicted mean temperature profiles compared with experimental data. 


\section{Figures:}

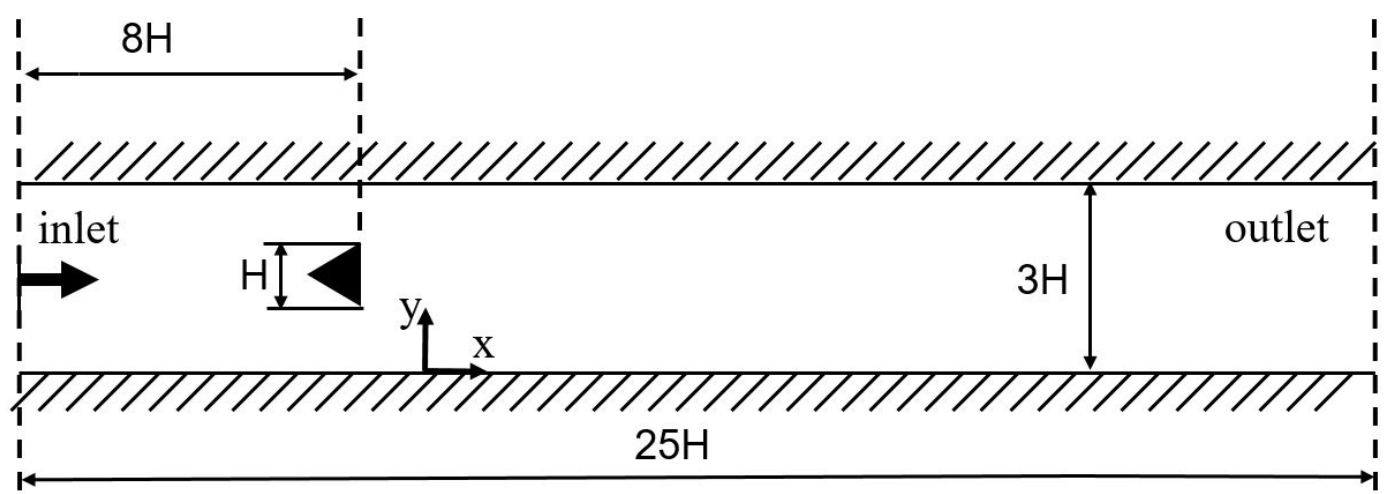

Figure 1. Schematic of the Volvo Rig combustor. The interior width in the z-direction is $6 H$.

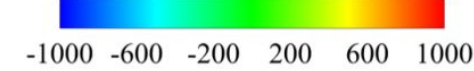

Z-Vorticity $(1 / \mathrm{s})$

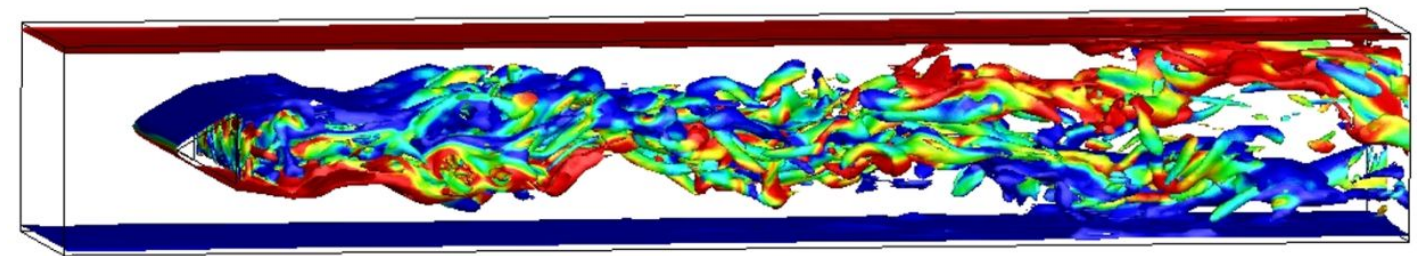

Figure 2. Iso-surface of vorticity magnitude level $2000 \mathrm{~s}^{-1}$ vorticity colored by the $\mathrm{z}$ component of vorticity.

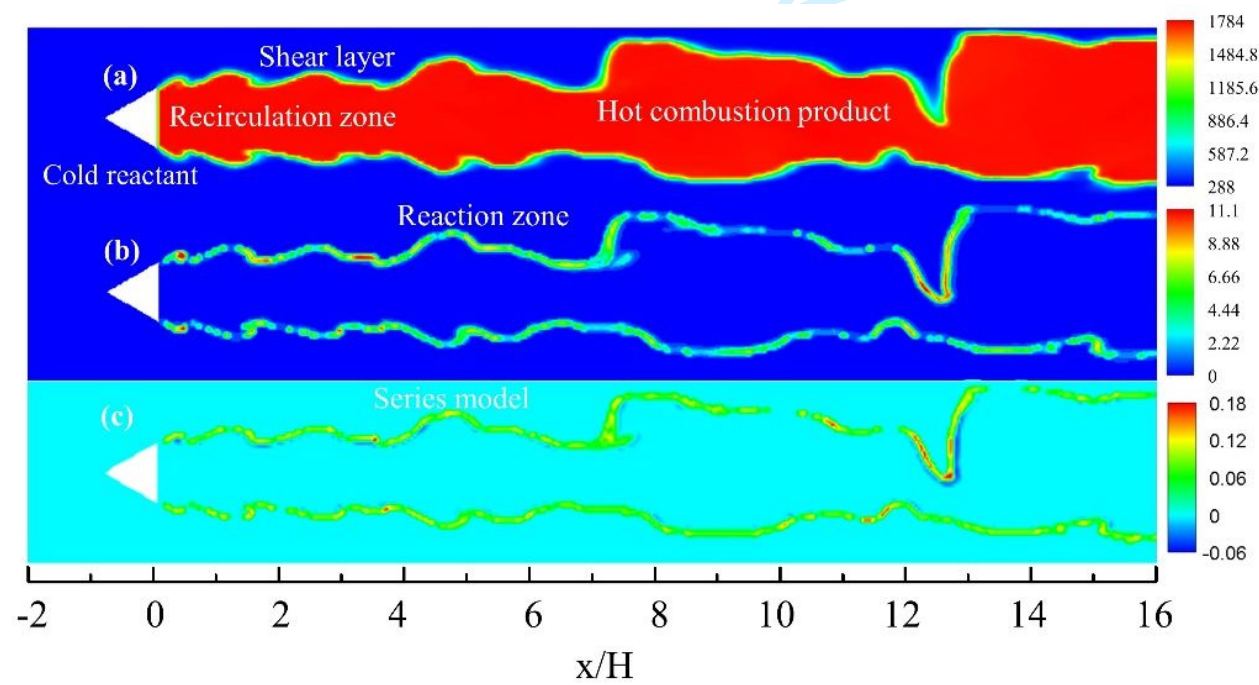

Figure 3. Instantaneous contours of $a$ ) temperature, $b$ ) absolute value of the $\mathrm{C}_{3} \mathrm{H}_{8}$ reaction rate $|\dot{\omega}(\bar{c})|$ and $c$ ) SGS contribution ratio of $\mathrm{C}_{3} \mathrm{H}_{8}$ reaction rate, $\dot{\omega} S G S / \dot{\omega}(\bar{c})$. 


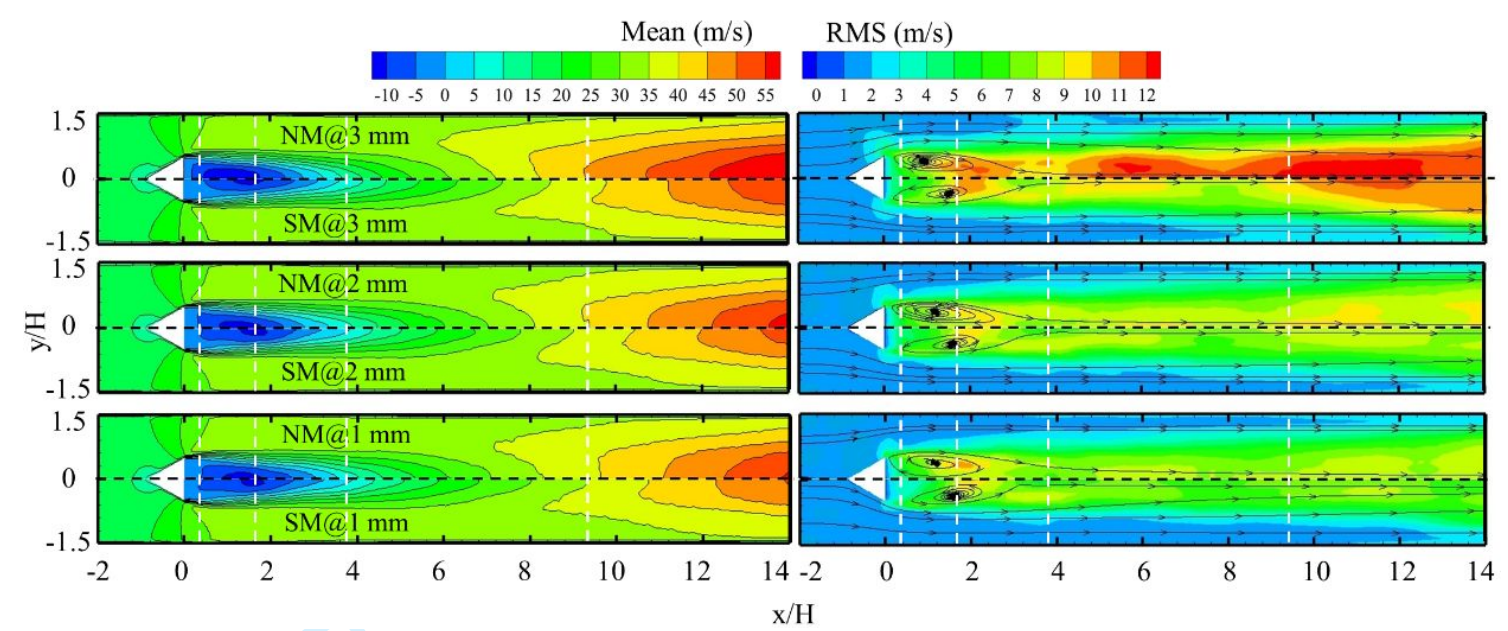

Figure 4. Mean (left) and RMS (right) axial velocity contours at different grid resolutions.

$\mathrm{NM}$ is short for no model approach and SM for series model. $1 \mathrm{~mm}, 2 \mathrm{~mm}$, and $3 \mathrm{~mm}$ stand for the grid resolutions. These abbreviations are also in effect in the following figures. Black lines in the left part represent the iso-contours of different mean velocity levels in the colour bar. Black lines with arrows stand for the stream traces of the mean velocity field.

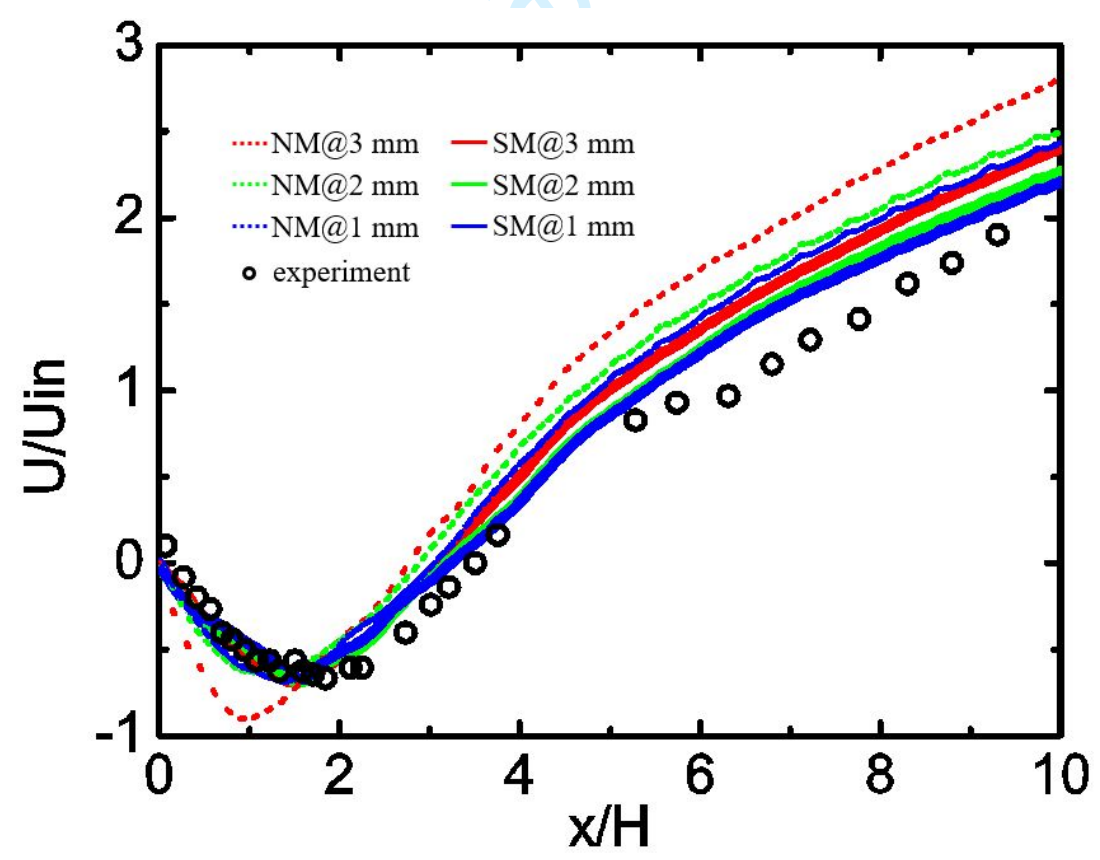

Figure 5. Centerline mean axial profile for different models in different grid resolutions 


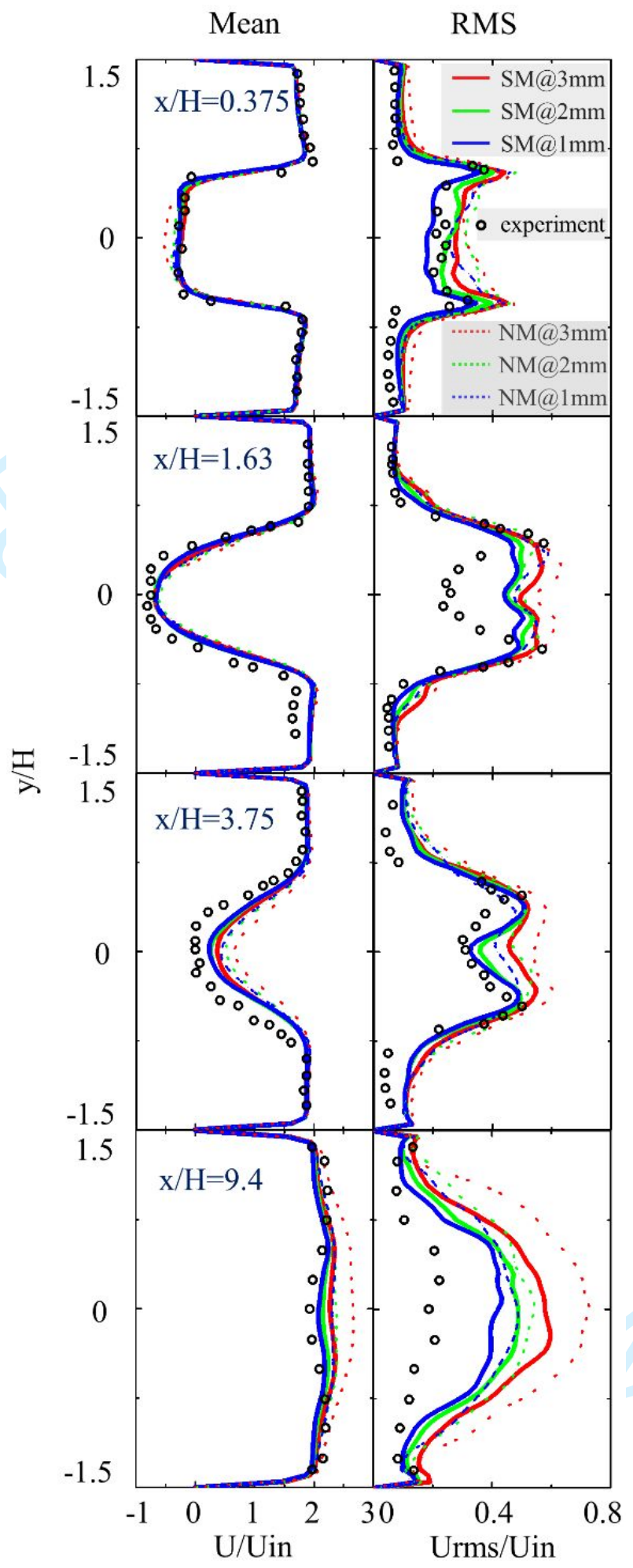

Figure 6. Mean and RMS axial velocity profiles for different models in different grid resolutions 


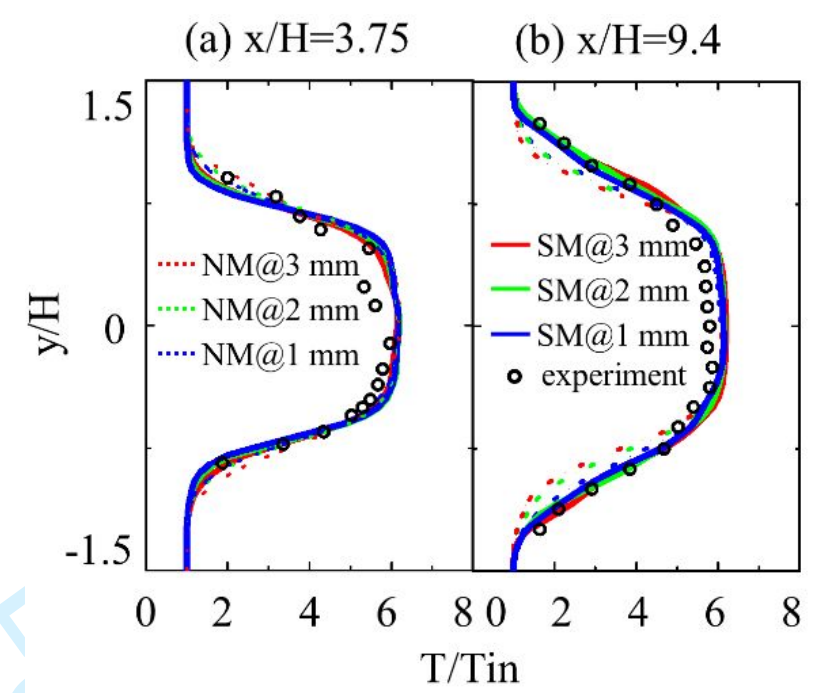

Figure 7. Predicted mean temperature profiles compared with experimental data. 